\title{
Periodic Motion in an Excited and Damped Cubic Nonlinear Oscillator
}

\author{
L. Cveticanin $\mathbb{D}^{1},{ }^{1,2}$ U. Kozmidis Luburic, ${ }^{1}$ and Gy. Mester ${ }^{3}$ \\ ${ }^{1}$ Faculty of Technical Sciences, University of Novi Sad, Novi Sad, Serbia \\ ${ }^{2}$ Doctoral School of Safety and Security, Obuda University, Budapest, Hungary \\ ${ }^{3}$ Faculty of Engineering, University of Szeged, Szeged, Hungary \\ Correspondence should be addressed to L. Cveticanin; cveticanin@uns.ac.rs
}

Received 14 March 2018; Accepted 7 May 2018; Published 28 June 2018

Academic Editor: Angelo Di Egidio

Copyright (C) 2018 L. Cveticanin et al. This is an open access article distributed under the Creative Commons Attribution License, which permits unrestricted use, distribution, and reproduction in any medium, provided the original work is properly cited.

\begin{abstract}
This paper investigates the steady-state periodic motion in the excited and damped one-degree-of-freedom Duffing oscillator. The oscillator is of the pure cubic type. The excitation is periodical and described by the product of two Jacobi elliptic functions. The mathematical model of the oscillator is a nonhomogeneous second-order strong nonlinear differential equation. The paper develops a procedure for obtaining the steady-state solution of the equation. Conditions for the existence of the steady-state motion of the oscillator are obtained. The influence of the excitation and of the damping on the steady-state motion is analyzed. The paper also investigates the transient to the steady-state motion in the parameter perturbed systems. An analytical method based on the time variable amplitude and the time variable phase is developed. The analysis of the obtained results shows that the damping parameter is an adequate control parameter for the steady-state motion of the oscillator. Analytically obtained results are compared with numerically obtained ones. The difference between solutions is negligible.
\end{abstract}

\section{Introduction}

Nowadays, there are a significant number of problems and devices which require the application of nonlinear oscillators with multifrequency excitation. Let us mention some of them.

For several microelectromechanical systems (MEMS), sensors, and actuators, the primary building blocks are microbeam resonators [1] excited with a multifrequency electrical source $[2,3]$ or different types of forces such as piezoelectric [4], electromagnetic [5], thermal [6], and electrostatic [7]. Based on piezoelectric multifrequency MEMS resonator the single-chip, which is applicable for wireless communications, is formed [8]. The multifrequency oscillator, with more than 600 summarized sine signals, is applied for rapid and accurate dynamic testing of micromachined gyroscope chips. Gyroscope chips are key components in micromachined gyroscopes (a type of angular rate sensors) widely used in military, automotive, consumer electronics, and other fields [9].

For diagnosing a variety of cardiovascular diseases, the ultrasound based velocimetry, termed echo particle image velocimetry (echo PIV), is developed [10]. The device uses ultrasonic multifrequency forcing (of the near rectangular type). The imaging is created on the basis of the mechanical response of tissue to multifrequency excitation [11]. Ultrasound radiation force is used to deform tissue, while the multiple ultrasound frequencies simultaneously produce multifrequency harmonic radiation force which is utilized for motion detection in vibrometry.

The atomic force microscope resonator also uses multifrequency excitation to generate high-resolution imaging and to extract the properties of the investigated surface $[12,13]$.

Generally, all of the abovementioned systems commonly have multiharmonic excitation, and they represent strong nonlinear oscillators. The multifrequency excitation force is a sum of various harmonic functions. For computational reasons and for the purpose of a more appropriate model analysis, we propose simplifying excitation by applying the product of the sine (sn) and delta $(\mathrm{dn})$ Jacobi elliptic functions [14]. In addition, let us assume that the nonlinearity of the oscillator is of a cubic type. In that case, the model which 
describes the previous devices is a periodically excited and damped Duffing oscillator.

Significant investigations in solving the strong nonlinear Duffing oscillator have been undertaken recently. For the free strong nonlinear Duffing oscillator the closed-form analytical solution is determined $[15,16]$. Various approximate solving methods are developed for the damped Duffing oscillator [17, 18]. For the harmonically excited strong nonlinear Duffing oscillator the approximate steady states are determined [19, 20]. Vakakis and Blanchard [21] were the first to calculate the exact steady states of the damped Duffing oscillator excited with the periodical force of the cosine Jacobi elliptic function form. Namely, they assumed that the excitation force and the steady-state functions have the same form as the free undamped Duffing oscillator, i.e., the form of the cosine (ca) Jacobi elliptic function. The method was introduced by Hsu [22] and extended by Rosenberg [23, 24] and recently by Rakaric et al. [25].

However, our intention is to generalize the problem by using another periodic excitation (not the mandatory cn) of the damped Duffing oscillator. The excitation force is assumed to be a multiperiodic function of the nearrectangular type. A procedure for obtaining the exact steadystate solution is developed. A constraint for the closed-form analytic solution is obtained. The relation between the excitation parameters and the damping coefficient (assumed as the control parameter of the system) is defined. The influence of the variation of the excitation, and of the damping coefficient on the steady-state motion of the oscillator is investigated. An analytical method for approximately solving the oscillator perturbed with a small damping force is developed. Based on the result, the prediction of the oscillator motion is available.

The paper has five sections. After the introduction, in Section 2, the explanation of the excitation force is given. The method for determination of the exact steady-state solution of the forced Duffing oscillator is developed. Conditions for steady-state periodic motion are defined. In Section 3, an analytical procedure for obtaining the periodical solution of the damped and excited strong nonlinear oscillator is developed. The method is specified for the case when perturbation is done with a viscous damping force. In Section 4, a numerical example is calculated. The analytically obtained solution is compared with a numerically obtained one.

\section{Exact Steady-State Solution}

Let us consider the steady-state motion of the excited Duffing oscillator. The Duffing oscillator has the strong cubic nonlinearity and the linear viscous damping. The excitation force is assumed to be a periodic function of almost rectangular type given in the Jacobi elliptic form

$$
F=F_{0} s n\left(\Omega t, k^{2}\right) d n\left(\Omega t, k^{2}\right),
$$

where $F_{0}$ is the amplitude of the excitation force and $s n$ and $d n$ are Jacobi elliptic functions [14] with frequency $\Omega$ and modulus $k^{2}$. The excitation (1) is periodical with period

$$
T=\frac{4 K(k)}{\Omega},
$$

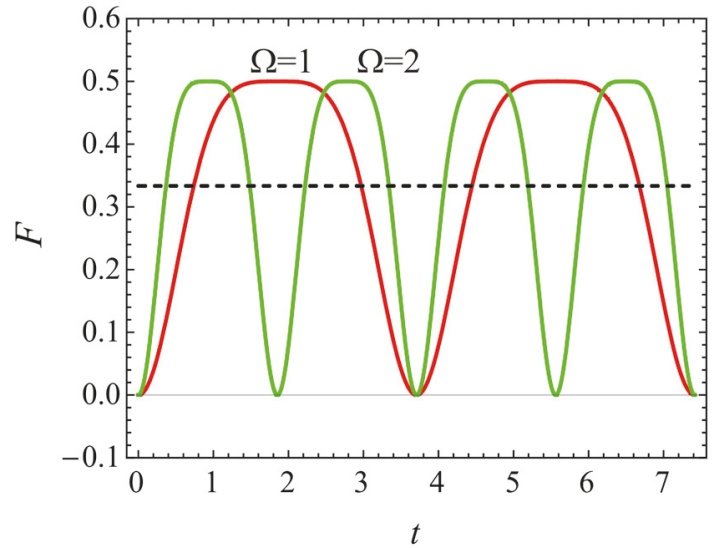

Figure 1: $F-t$ diagrams for $\Omega=1$ and $\Omega=2$.

where $K(k)$ is the complete elliptic integral of the first kind. In Figure 1 the excitation force for $F_{0}=1, k^{2}=1$ and various values of $\Omega$ is plotted.

It can be seen that the period of vibration is longer for smaller values of the frequency parameter $\Omega$. The excitation force (1) is often applied in signal processing but also in echo particle image velocimetry [10]. For the excitation force (1) the model of the damped Duffing oscillator is

$$
\ddot{x}+c_{3} x^{3}+b \dot{x}+F_{0} \operatorname{sn}\left(\Omega t, k^{2}\right) d n\left(\Omega t, k^{2}\right)=0,
$$

where $c_{3}$ is the coefficient of rigidity and $b$ is the damping coefficient. If the excitation and the damping are neglected the model of the free Duffing oscillator simplifies to

$$
\ddot{x}+c_{3} x^{3}=0 .
$$

Equation (4) has the exact solution

$$
x=\operatorname{Acn}\left(\Omega t, k^{2}\right)
$$

where $c n$ is the cosine Jacobi elliptic function and $A$ is the amplitude of vibration. Comparing the excitation force (1) and the solution of the free Duffing oscillator (5) it is evident that they are different functions and do not satisfy the criteria of Vakakis and Blanchard [21]. Because of that, we develop a new procedure for obtaining of the criteria for the exact steady-state solution of (3).

We assume the solution of (3) in the form of the cosine Jacobi elliptic function (5) which is the solution of the free undamped oscillator (4). Substituting (5) and its derivatives

$$
\dot{x}=-A \Omega s n\left(\Omega t, k^{2}\right) d n\left(\Omega t, k^{2}\right),
$$

and

$$
\ddot{x}=-A \Omega^{2} c n\left(\Omega t, k^{2}\right)\left(1-2 k^{2}+2 k^{2} c n^{2}\left(\Omega t, k^{2}\right)\right),
$$


into (3) and separating the terms with the same functions $c n\left(\Omega t, k^{2}\right), c^{3}\left(\Omega t, k^{2}\right)$, and $\operatorname{sn}\left(\Omega t, k^{2}\right) d n\left(\Omega t, k^{2}\right)$ the following algebraic equations are obtained:

$$
\begin{aligned}
-\Omega^{2}+c_{3} A^{2} & =0, \\
1-2 k^{2} & =0, \\
-b \Omega A+F_{0} & =0 .
\end{aligned}
$$

For the known excitation parameters $F_{0}$ and $\Omega$ and $k^{2}=1 / 2$, the amplitude of vibration and the damping coefficient for the steady state are as follows:

$$
\begin{aligned}
& A=\Omega \sqrt{c_{3}}, \\
& b=\frac{F_{0}}{\sqrt{c_{3}} \Omega^{2}} .
\end{aligned}
$$

The coefficient of damping $b$ depends directly on the amplitude and indirectly on the frequency of the excitation. Besides, the steady-state amplitude $A$ depends on the frequency of the excitation function and does not depend on the amplitude of the excitation.

Using this result, we suggest controlling the periodicity of the system by changing of the damping coefficient. If the amplitude of excitation force is increasing, the damping in the system has to be increased according to $(9)_{2}$, while the amplitude and frequency of vibration of the system remain unchanged.

If the frequency of excitation is changed, it is necessary to vary the damping coefficient according to $(9)_{2}$ : decrease of the frequency of excitation causes increase of the damping coefficient. For that case, the motion is periodic, but the amplitude of vibration is varying: decrease of frequency causes decrease of the amplitude of vibration, too.

As an example, let us consider an oscillator described with

$$
\ddot{x}+x^{3}+\dot{x}+\operatorname{sn}\left(t, \frac{1}{2}\right) d n\left(t, \frac{1}{2}\right)=0,
$$

where $F_{0}=1, \Omega=1$, and $c_{3}=1$ and the coefficient of damping is according to $(9)_{2}$

$$
b=1 .
$$

The analytical solution for the steady-state motion is

$$
x=c n\left(t, \frac{1}{2}\right) \text {. }
$$

Using the Runge-Kutta procedure (10) is solved numerically for the initial conditions $x(0)=0$ and $\dot{x}(0)=0$. In Figure 2 the analytical solution (12) and the numerical solution of (10) are compared.

It can be seen that the results agree.

\section{Perturbed Steady-State Motion}

Let us consider the weakly perturbed steady-state oscillator. Mathematical model of the system is

$$
\begin{aligned}
\ddot{x} & +c_{3} x^{3}+b \dot{x}+F_{0} \operatorname{sn}\left(\Omega t, k^{2}\right) d n\left(\Omega t, k^{2}\right) \\
& =\varepsilon f(x, \dot{x}),
\end{aligned}
$$

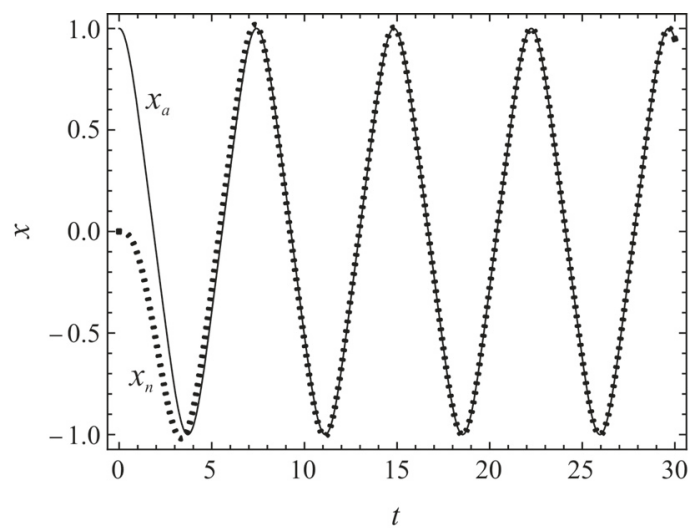

FIGURE 2: $x_{a}-t$ analytically (full line) and $x_{n}-t$ numerically (dotted line) obtained diagram for $b=1$.

where $\varepsilon \quad<1$ is a small parameter and $f(x, \dot{x})$ is the perturbation function. A method for approximate solving of (13) is developed. The method is based on the assumption that the solution of the perturbed equation (13) has to be the perturbed version of the nonperturbed solution (5) of (3). Namely, the solution and its derivative are assumed in the form of (5), but with time variable amplitude and phase

$$
x=A(t) c n\left(\psi(t), \frac{1}{2}\right),
$$

and

$$
\dot{x}=-A(t) \omega s n\left(\psi(t), \frac{1}{2}\right) d n\left(\psi(t), \frac{1}{2}\right),
$$

where

$$
\dot{\psi}(t)=\omega+\dot{\theta}(t),
$$

and $\omega$ is equal to $\Omega$ for $f(x, \dot{x})=0$. Otherwise, the time derivative of the solution (14) is

$$
\begin{aligned}
\dot{x}= & -A(t)(\omega+\theta(t)) s n\left(\psi(t), \frac{1}{2}\right) d n\left(\psi(t), \frac{1}{2}\right) \\
& +A(t) c n\left(\psi(t), \frac{1}{2}\right) .
\end{aligned}
$$

Comparing (15) and (17) and equating the expressions the following constraint is obtained:

$$
\dot{A} c n-A \dot{\theta} s n d n=0,
$$

where $(\psi(t), 1 / 2)=s n, c n(\psi(t), 1 / 2)=c n$, and $d n(\psi(t), 1 / 2)=d n$. Substituting (14), (15), and its derivative into (13) we have

$$
-(\dot{A} \omega+A \dot{\omega}) s n d n-A \omega \dot{\theta} c n^{3}=\varepsilon f(A c n,-A \omega s n d n) .
$$

Differentiating the relation $(9)_{1}$ for time variable amplitude and frequency, we have

$$
\dot{A} \omega=A \dot{\omega} .
$$


Using (20), (19) transforms into

$$
-2 \dot{A} \omega s n d n-A \omega \dot{\theta} c n^{3}=\varepsilon f(A c n,-A \omega s n d n) .
$$

Equations (18) and (21) correspond to (13). After some modification the equations of motion of the oscillator are

$$
\begin{aligned}
\dot{A} \omega & =-\varepsilon f(A c n,-A \omega s n d n) s n d n, \\
A \omega \dot{\theta} & =-\varepsilon f(A c n,-A \omega s n d n) c n .
\end{aligned}
$$

These are two coupled first-order equations. To solve them is not an easy task. It is at this point that the averaging is introduced. The averaging is over the period of the Jacobi elliptic functions. The averaged equations are

$$
\begin{aligned}
\dot{A} \omega & =-\langle\varepsilon f(A c n,-A \omega s n d n) s n d n\rangle, \\
A \omega \dot{\theta} & =-\langle\varepsilon f(A c n,-A \omega s n d n) c n\rangle,
\end{aligned}
$$

where $\langle\bullet\rangle=(1 / T) \int_{0}^{T}(\bullet) d \psi$. Solving (24) and (25) the approximate averaged solutions of equations are obtained.

\section{Perturbation of the Damping Force}

As the special case, the oscillator perturbed with linear viscous damping force is considered. This phenomenon occurs during steady-state motion control by varying of the damping coefficient. For that case the coefficient of damping is

$$
b_{1}=b+b_{2} \text {, }
$$

where $b_{2}<<b$ is a small perturbation damping parameter. The equation of motion is

$$
\ddot{x}+c_{3} x^{3}+b \dot{x}+F_{0} s n\left(\Omega t, \frac{1}{2}\right) d n\left(\Omega t, \frac{1}{2}\right)=-b_{2} \dot{x} .
$$

It is the perturbed version of (3). Using the suggested procedure and the relation $(9)_{2}$ we transform (27) into

$$
\begin{aligned}
\dot{A} & =-\left(A b_{1}-\frac{F_{0}}{\Omega}\right) s n^{2} d n^{2}, \\
A \dot{\theta} & =-\left(A b_{1}-\frac{F_{0}}{\Omega}\right) \text { sncndn. }
\end{aligned}
$$

For

$$
\begin{aligned}
\frac{1}{T} \int_{0}^{T} s n c n d n d \psi & =0 \\
\left\langle s n^{2} d n^{2}\right\rangle & =\frac{1}{T} \int_{0}^{T} s n^{2} d n^{2} d \psi,
\end{aligned}
$$

the averaged equations are

$$
\begin{aligned}
\dot{A}+b_{1} A\left\langle s n^{2} d n^{2}\right\rangle & =\frac{F_{0}}{\Omega}\left\langle s n^{2} d n^{2}\right\rangle, \\
\dot{\theta} & =0 .
\end{aligned}
$$

The solution of the uncoupled first-order differential equation (31) is

$$
A=\frac{F_{0}}{\omega b_{1}}+C \exp \left(-b_{1}\left\langle s n^{2} d n^{2}\right\rangle t\right),
$$

where $C$ is the constant of integration which satisfies the relation for initial amplitude $(8)_{3}$

$$
A_{0}=\frac{F_{0}}{\Omega b} \text {. }
$$

Finally,

$$
A=\frac{F_{0}}{\omega b_{1}}+\left(A_{0}-\frac{F_{0}}{\omega b_{1}}\right) \exp \left(-b_{1}\left\langle s n^{2} d n^{2}\right\rangle t\right) .
$$

For the steady-state case, when $t \longrightarrow \infty$, the amplitude of vibration tends to

$$
A_{\rightarrow \infty}=\frac{F_{0}}{\omega b_{1}} .
$$

The amplitude has an influence on the frequency of vibration. Namely, substituting (36) into (9) it is

$$
\omega=\sqrt{c_{3}} A \rightarrow \infty .
$$

Solving (36) and (37) the steady-state amplitude and frequency of the Jacobi elliptic function yield

$$
\begin{aligned}
A_{\rightarrow \infty} & =\sqrt{\frac{F_{0}}{b_{1} \sqrt{c_{3}}}}, \\
\omega & =\sqrt{\frac{F_{0} \sqrt{c_{3}}}{b_{1}}} .
\end{aligned}
$$

Integrating the relation (32) and using (16) and (38) the steady-state oscillatory motion is

$$
x=\sqrt{\frac{F_{0}}{b_{1} \sqrt{c_{3}}}} c n\left(t \sqrt{\frac{F_{0} \sqrt{c_{3}}}{b_{1}}}, \frac{1}{2}\right) .
$$

The properties of the oscillatory motion depend on the intensity of excitation, coefficient of rigidity of the oscillator, and the damping: parameter. For higher value of excitation the amplitude and the frequency of vibration are higher. For the oscillator with higher rigidity the amplitude of vibration is smaller, while the frequency of vibration is higher. The damping coefficient acts indirectly on the vibration amplitude and frequency: the higher the damping, the smaller the amplitude and also the frequency of oscillatoxin.

Comparing the amplitude and frequency (38) with the initial amplitude (34) and frequency

$$
\begin{aligned}
A_{0} & =\frac{1}{\sqrt[4]{c_{3}}}\left(\frac{F_{0}}{b}\right)^{1 / 2}, \\
\Omega & =\sqrt[4]{c_{3}}\left(\frac{F_{0}}{b}\right)^{1 / 2},
\end{aligned}
$$

it can be concluded that the amplitude and the frequency have the tendency of decrease with increasing of the damping coefficient, and the period of vibration increases. 


\section{Example}

Let us consider the perturbed steady-state oscillator

$$
\ddot{x}+x^{3}+1.1 \dot{x}=-s n\left(t, \frac{1}{2}\right) d n\left(t, \frac{1}{2}\right) \text {. }
$$

Namely, rewriting (41) we have

$$
\ddot{x}+x^{3}+\dot{x}+s n\left(t, \frac{1}{2}\right) d n\left(t, \frac{1}{2}\right)=-0.1 \dot{x},
$$

where the term on the right side represents the perturbation for the steady-state oscillator described with the equation

$$
\ddot{x}+x^{3}+\dot{x}+s n\left(t, \frac{1}{2}\right) d n\left(t, \frac{1}{2}\right)=0 .
$$

The analytical solution of (43) is already given (12) and plotted.

Using the procedure, given in the previous section, the analytical solution of (41) is obtained as

$$
x_{a}=\left(0.9+(1-0.9) \exp \left(-\frac{1.1 t}{6}\right)\right) c n\left(0.985 t, \frac{1}{2}\right),
$$

while the steady-state solution is

$$
x_{a}=0.9 c n\left(0.985 t, \frac{1}{2}\right) .
$$

For initial conditions $x(0)=0$ and $\dot{x}(0)=0$ (41) is solved also numerically. In Figure 3 the analytically and numerically obtained results are compared. From Figure 3 it is obvious that the analytical solution is in a good agreement with numerical one. In Figure 4 the $x-t$ diagrams obtained for $b=1$ and $b_{1}=1.1$ are compared. It is seen that the amplitude of vibration is lower for the case when $b_{1}>b$ while the period of vibration is longer.

\section{Conclusion}

This paper investigates the exact steady-state solution for the forced and damped Duffing oscillator. The steady-state solution is assumed in the form which corresponds to the steady-state motion of the free undamped Duffing oscillator. Mathematically, it is described with the cosine Jacobi elliptic function. The condition for the existence of the exact steadystate solution is determined. The analysis concludes that for a certain relation between the damping coefficient and the excitation parameters the steady-state motion of the system is permanent. However, if excitation parameters are varied, the steady-state motion is perturbed. To force the system to move periodically after perturbation, the damping coefficient of the oscillator has to be varied. Thus, if the amplitude of the excitation force is increased, the damping coefficient of the system has to be increased as well. If the frequency of excitation is varied, the damping coefficient has to be changed up to the value which gives the steady-state motion.

The position of the steady-state solution can be controlled by changing the damping coefficient. Varying the damping

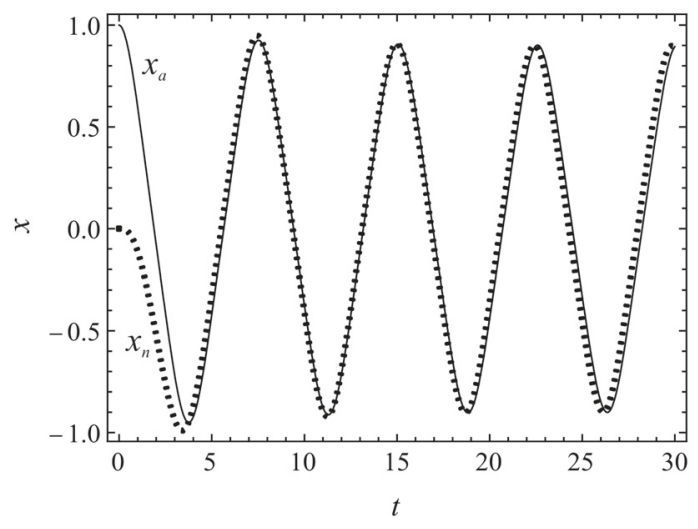

FIGURE 3: $x_{a}-t$ analytically (full line) and $x_{n}-t$ numerically (dotted line) obtained diagrams for $b=1.1$.

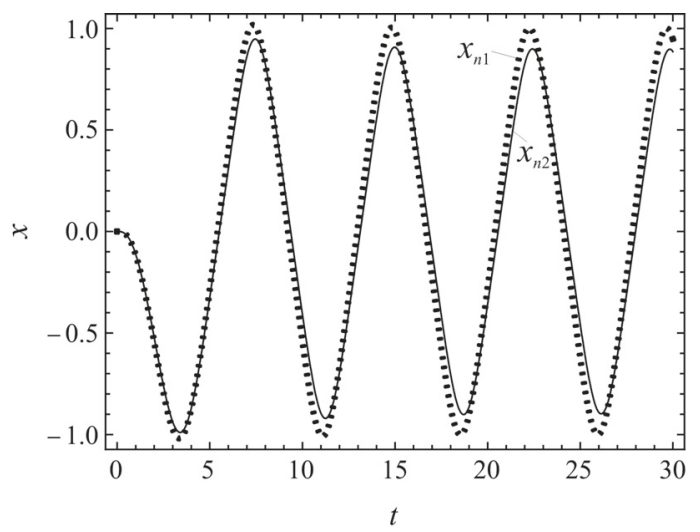

FIgURE 4: $x_{n 1}-t$ diagram for $b=1$ (dotted line) and $x_{n 2}-t$ diagram for $b_{1}=1.1$ (full line).

coefficient, we force the system to have the periodical steadystate motion which corresponds to the free undamped Duffing oscillator. If the variation of the damping coefficient is small, the transient motion of the oscillator lasts for a short time and the stable steady-state motion follows. Based on this result, we conclude that the damping coefficient is an appropriate control parameter for the steady-state motion for the forced and damped Duffing oscillator. We recommend the application of the result for a new control technique in nonlinear oscillators.

\section{Data Availability}

No data were used to support this study.

\section{Conflicts of Interest}

The authors declare that they have no conflicts of interest.

\section{Acknowledgments}

This investigation is supported by the Faculty of Technical Sciences in Novi Sad, Serbia (Proj. no. 054). 


\section{References}

[1] Z. Saadatnia, H. Askari, and E. Esmailzadeh, "Multi-frequency excitation of microbeams supported by Winkler and Pasternak foundations," Journal of Vibration and Control, pp. 1-18, 2017.

[2] N. Jaber, A. Ramini, and M. I. Younis, "Multifrequency excitation of a clampedclamped microbeam: Analytical and experimental investigation," Microsystems and Nanoengineering, vol. 2, article 16002, 6 pages, 2016.

[3] N. Jaber, A. Ramini, Q. Hennawi, and M. I. Younis, "Wideband MEMS resonator using multifrequency excitation," Sensors and Actuators A: Physical, vol. 242, pp. 140-145, 2016.

[4] B. Piekarski, M. Dubey, and D. Devoe, "Fabrication of suspended piezoelectric Microresonators," Integrated Ferroelectrics, vol. 24, pp. 147-154, 1999.

[5] D. Jin, X. Li, J. Liu et al., "High-mode resonant piezoresistive cantilever sensors for tens-femtogram resoluble mass sensing in air," Journal of Micromechanics and Microengineering, vol. 16, no. 5, pp. 1017-1023, 2006.

[6] G. Rinaldi, M. Packirisamy, and I. Stiharu, "Quantitative boundary support characterization for cantilever MEMS," Sensors, vol. 7, no. 10, pp. 2062-2079, 2007.

[7] A. Ramini, M. I. Younis, and Q. T. Su, "A low-g electrostatically actuated resonant switch," Smart Materials and Structures, vol. 22, no. 2, p. 025006, 2013.

[8] C. Zuo, N. Sinha, J. Van Der Spiegel, and G. Piazza, "Multi-frequency pierce oscillators based on piezoelectric AlN contour-mode MEMS resonators," in Proceedings of the International Frequency Control Symposium, FCS '08, pp. 402-407, IEEE, May 2008.

[9] Y. Deng, B. Zhou, C. Xing, and R. Zhang, "Multifrequency excitation method for rapid and accurate dynamic test of micromachined gyroscope chips," Sensors, vol. 14, no. 10, pp. 19507-19516, 2014.

[10] H. Zheng, O. Mukdadi, H. Kim, J. R. Hertzberg, and R. Shandas, "Advantages in using multifrequency excitation of contrast microbubbles for enhancing echo particle image velocimetry techniques: Initial numerical studies using rectangular and triangular waves," Ultrasound in Medicine \& Biology, vol. 31, no. 1, pp. 99-108, 2005.

[11] M. W. Urban, M. Fatemi, and J. F. Greenleaf, "Modulation of ultrasound to produce multifrequency radiation force," The Journal of the Acoustical Society of America, vol. 127, no. 3, pp. 1228-1238, 2010.

[12] R. Jose, L. Garcia, and R. Garcia, "Theory of multifrequency atomic force microscopy," Physical Review Letters, vol. 100, Article ID 076102, 2008.

[13] D. Forchheimer, D. Platz, and E. A. Tholén, "Model-based extraction of material properties in multifrequency atomic force microscopy," Physical Review B, vol. 85, Article ID 195449, 2012.

[14] P. F. Byrd and M. D. Friedman, Handbook of Elliptic Integrals for Engineers and Physicists, Springer, Berlin, Germany, 1954.

[15] L. Cveticanin, Strongly Nonlinear Oscillators-Analytical Solutions, Undergraduate Lecture Notes in Physics, Springer, 2014.

[16] L. Cveticanin, "Pure nonlinear oscillator," in Strong Nonlinear Oscillators, Mathematical Engineering, pp. 17-49, Springer, 2018, 9783319588254.

[17] L. Cveticanin, "The approximate solving methods for the cubic Duffing equation based on the Jacobi elliptic functions," International Journal of Nonlinear Sciences and Numerical Simulation, vol. 10, no. 11-12, pp. 1491-1516, 2009.
[18] L. Cveticanin, "Analyses of oscillators with non-polynomial damping terms," Journal of Sound and Vibration, vol. 317, no. 3-5, pp. 866-882, 2008.

[19] L. Cveticanin, G. Mester, and I. Biro, "Parameter influence on the harmonically excited Duffing oscillator," Acta Polytechnica Hungarica, vol. 11, no. 5, pp. 145-160, 2014.

[20] I. Kovacic, L. Cveticanin, M. Zukovic, and Z. Rakaric, "Jacobi elliptic functions: a review of nonlinear oscillatory application problems," Journal of Sound and Vibration, vol. 180, pp. 1-36, 2016.

[21] A. F. Vakakis and A. Blanchard, "Exact steady states of the periodically forced and damped Duffing oscillator," Journal of Sound and Vibration, vol. 413, pp. 57-65, 2018.

[22] C. S. Hsu, "On the application of elliptic functions in non-linear forced oscillations," Quarterly of Applied Mathematics, vol. 17, pp. 393-407, 1959/1960.

[23] R. M. Rosenberg, "On nonlinear vibrations of systems with many degrees of freedom," Advances in Applied Mechanics, vol. 9, no. C, pp. 155-242, 1966.

[24] R. M. Rosenberg, "Steady-state forced vibrations," International Journal of Non-Linear Mechanics, vol. 1, no. 2, pp. 95-108, 1966.

[25] Z. Rakaric, I. Kovacic, and M. Cartmell, "On the design of external excitations in order to make nonlinear oscillators respond as free oscillators of the same or different type," International Journal of Non-Linear Mechanics, vol. 94, pp. 323333, 2017 


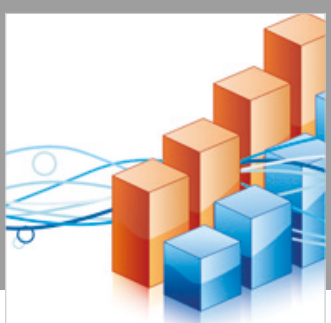

Advances in

Operations Research

\section{-n-m}
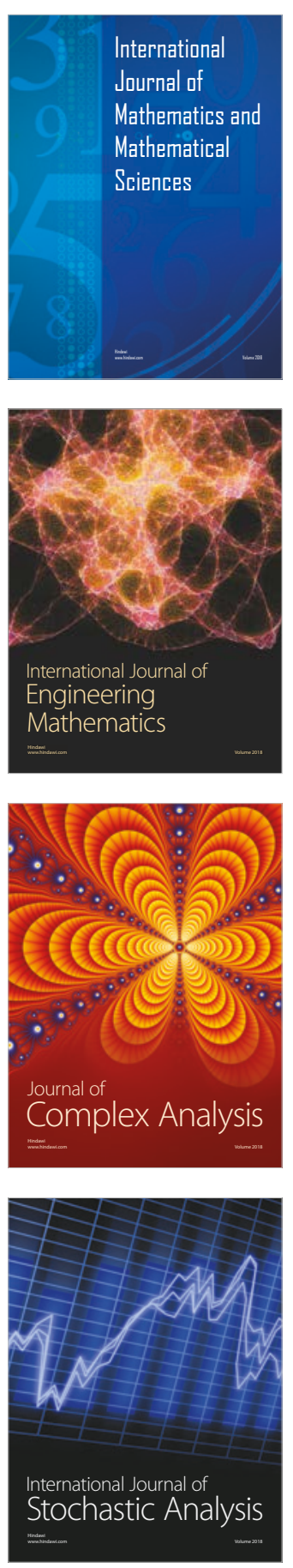
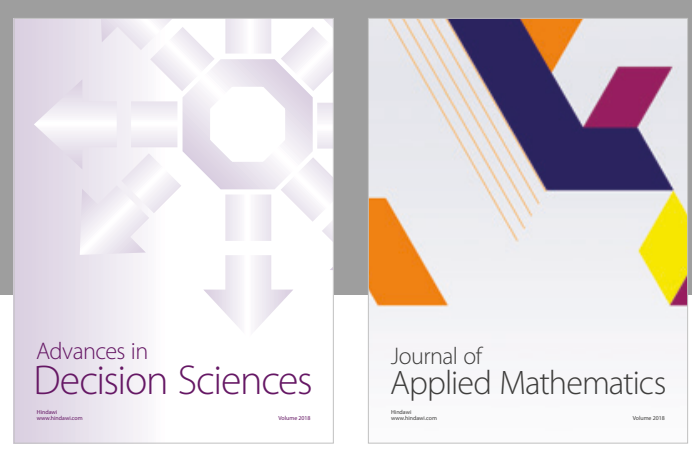

Journal of

Applied Mathematics
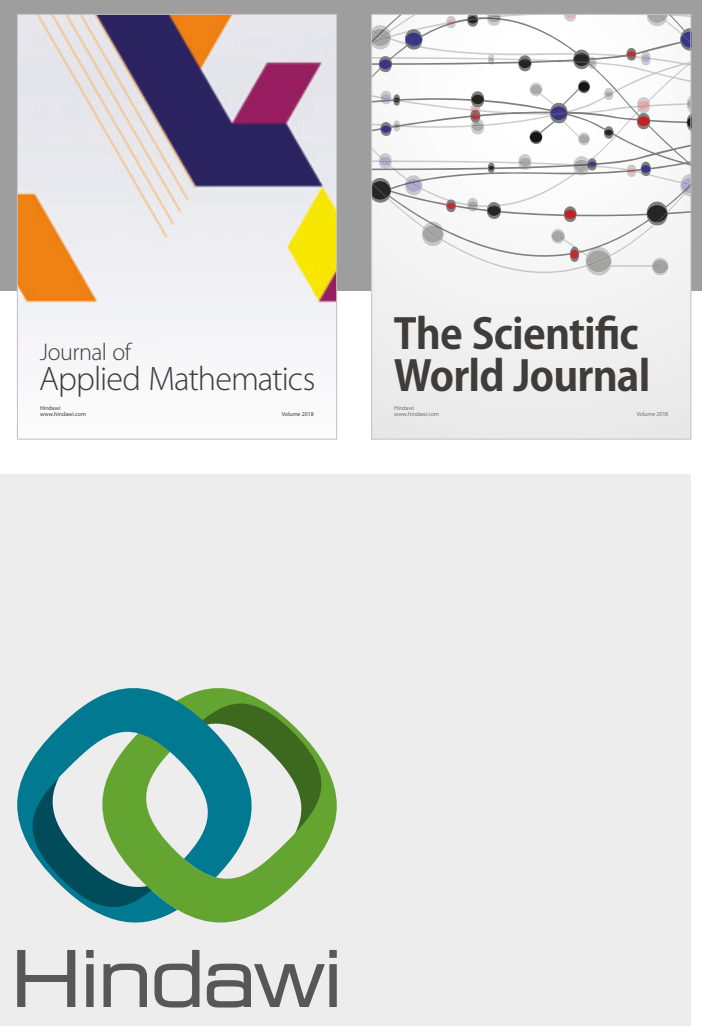

Submit your manuscripts at

www.hindawi.com

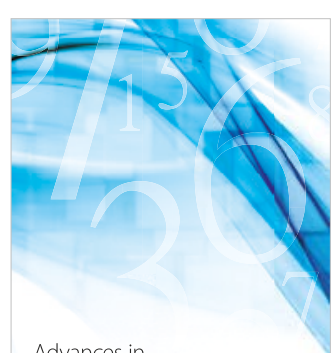

Advances in
Numerical Analysis
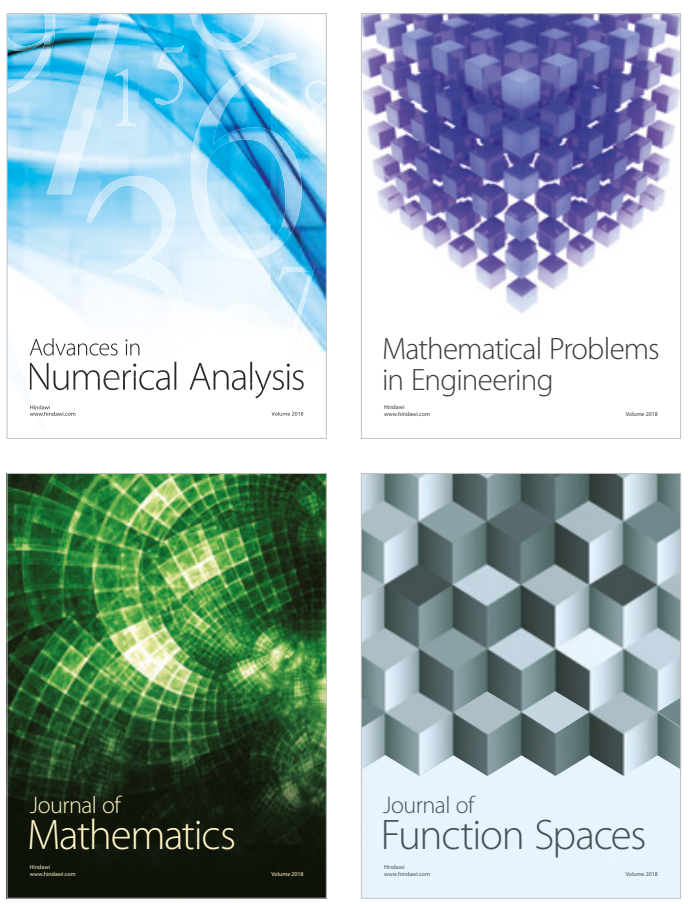

Mathematical Problems in Engineering

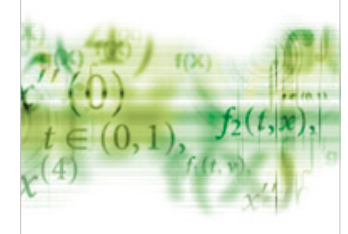

International Journal of

Differential Equations

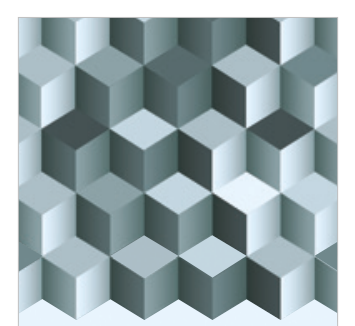

Journal of

Function Spaces

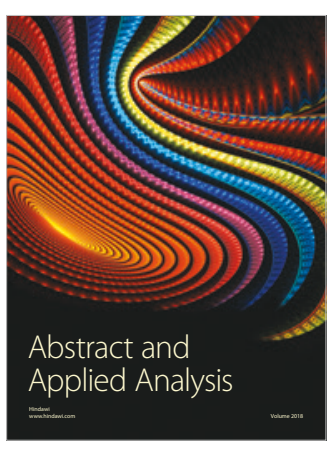

The Scientific

World Journal

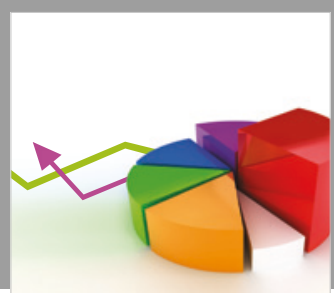

Journal of

Probability and Statistics
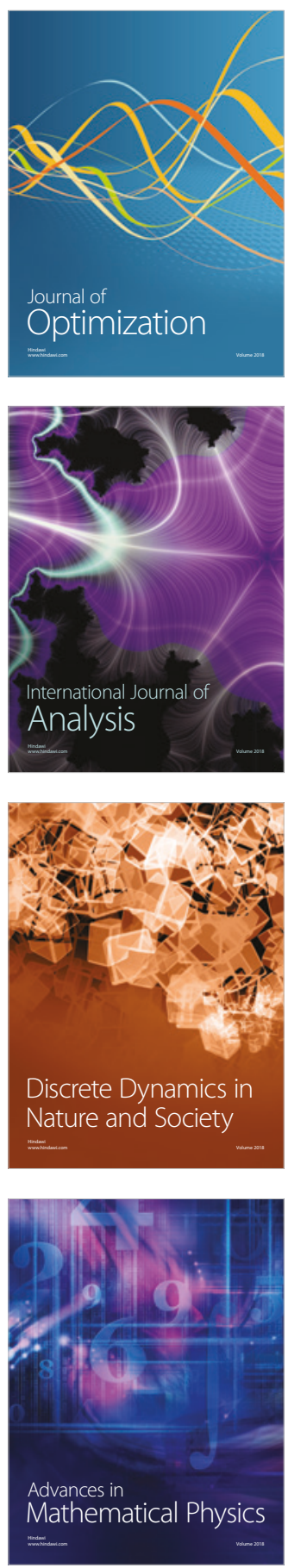